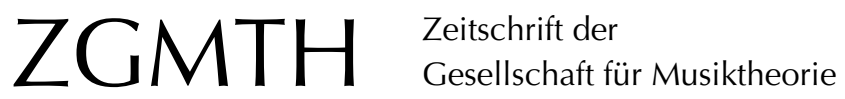

Bauer, Cornelius (2006): "What power art thou?«. Zur Harmonik Henry Purcells am Beispiel der Arie des Cold Genius aus King Arthur. ZGMTH 3/3, 327-340.

https://doi.org/10.31751/238

(C) 2006 Cornelius Bauer

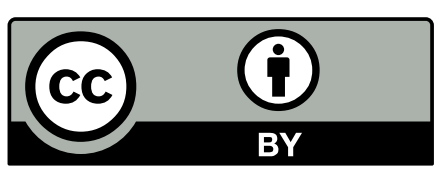

Dieser Text erscheint im Open Access und ist lizenziert unter einer Creative Commons Namensnennung 4.0 International Lizenz.

This is an open access article licensed under a Creative Commons Attribution 4.0 International License.

veröffentlicht / first published: 01/07/2006 zuletzt geändert / last updated: 01/12/2008 


\section{»What power art thou?»

\author{
Zur Harmonik Henry Purcells am Beispiel der Arie des Cold Genius \\ aus King Arthur
}

Cornelius Bauer

Henry Purcell überrascht immer wieder durch ungewöhnliche harmonische Wendungen, so auch in der Arie "What power art thou " des Cold Genius aus seiner Oper King Arthur (1691): Bereits das Orchestervorspiel enthält einige Besonderheiten, die sich weder mit Hilfe einer funktionstheoretischen Analyse noch durch den Rekurs auf zeitgenössische Generalbassmodelle restlos erklären lassen. In Anbetracht der konsequent linear geführten Singstimme wird der harmonische Verlauf des Vorspiels auf zwei Strukturstimmen zurückgeführt, die sich als 'Querschnitt der realen Stimmverläufe im Orchestersatz bilden lassen. Als thematisch wirksame Elemente lassen sie sich durch die ganze Arie hindurch sowohl in der Singstimme als auch im Orchestersatz nachweisen.

\section{I}

Der Komponist Henry Purcell (1659-95) gehört trotz aller gegenteiligen Bemühungen nach wie vor zu jenen Komponisten, die im Konzertleben und mehr noch in der Musikwissenschaft eine eher nachgeordnete Rolle spielen. Dabei, so zeigt der ihm zuerkannte Titel »Orpheus Britannicus «", war er zu Lebzeiten durchaus erfolgreich und hochgeschätzt. In der Folge jedoch kam sein Werk allmählich aus der Mode und versank in Vergessenheit, bis man im 19. Jahrhundert, im Zuge des aufkommenden Nationalismus und Historismus, in Großbritannien begann, sich auf den heimischen Meister zu besinnen. ${ }^{2}$ Außerhalb seiner Heimat hatte es Purcells Musik lange Zeit schwer, sich gegen die zeitgenössische italienische, deutsche und französische Konkurrenz durchzusetzen. Erst in jüngerer Zeit hat die Alte-Musik-Bewegung Purcell wieder für sich entdeckt: Galt er zunächst noch als Geheimtip, avancierte er bald zum Modekomponisten diverser Ensembles und Veranstalter. In der wissenschaftlichen Diskussion jedoch findet er hierzulande nach wie vor nicht die Beachtung, die er eigentlich verdient.

Ein Grund könnte darin bestehen, dass Purcells Musik der Forschung zu wenig als Exempel gilt. Anders als die klare und einfach nachvollziehbare Musiksprache der italienischen Komponisten um Arcangelo Corelli erlangte die tendenziell unberechenbarere Musik Purcells keinen Modellcharakter für die europäische Musik des Hochba-

1 So der Titel einer posthum erschienenen Sammlung von Purcells Liedern.

2 Purcell Club 1836-1863, Gründung der Purcell Society 1876. 
rock. Dies betrifft insbesondere die Harmonik: Stützt sich Corelli weitgehend auf Sequenzmodelle, die durch die Verbreitung seiner Werke endgültig zum Standard der Zeit wurden, so bevorzugt Purcell demgegenüber eine heterogene Mischung aus teils konventionellen, dann wiederum höchst ungewöhnlichen Klangfortschreitungen.

Ein Beispiel hierfür ist die Arie "What power art thou« des Cold Genius aus der Oper King Arthur von 1691. Diese >Dramatick Opera', eine Art Mischung aus Schauspiel und Oper, behandelt in loser Szenenfolge die Auseinandersetzung zwischen dem britischen König Artus und dem Sachsenfürsten Oswald, der mit allerlei magischen Tricks seines Zauberers Osmond die Herrschaft über Britannien zu gewinnen sucht. Die hier betrachtete Arie stammt aus der sogenannten `Frost Scene`, einer reichlich fantastischen Demonstration Osmonds, in der der Sieg der Liebe über den Winter geschildert wird; hiermit soll die sfrostige` Jungfrau Esmeralda, Artus' Braut, diesem abspenstig gemacht werden. Offensichtlich ist diese Rahmenhandlung für die musikalische Gestaltung der Szene völlig unerheblich, Purcell setzt vielmehr das Bild einer im Eise erstarrten Winterlandschaft musikalisch um, aus der sich der skalte Geistı auf den Ruf Cupidos hin zitternd und widerstrebend erhebt.

\section{II}

Zunächst sei das Orchestervorspiel einer eingehenden Untersuchung unterzogen, da es durchaus modellhaften Charakter für die gesamte Arie hat. Die einfache homorhythmische Satzstruktur in repetierten Achteln, die einen zumeist in Halben fortschreitenden Akkordsatz bilden, lassen harmonische Besonderheiten deutlich hervortreten. Schon beim ersten Hören fallen einige ungewohnte Wendungen auf: Da wäre zunächst der merkwürdige Sextvorhalt in Takt 3 zu nennen, den Purcell weder vorbereitet noch im Durchgang, sondern als betonte Wechselnote einführt. Der so entstehende übermäßige Dreiklang ist eines der von Purcell häufig gebrauchten harmonischen Ausdrucksmittel. Auch der Quintsextakkord auf der letzten Zählzeit mag aus stimmführungstechnischer Sicht verwundern, da der dissonierende Ton es ${ }^{1}$ ebenfalls nicht als Durchgang erscheint, sondern von unten angesprungen wird. ${ }^{3}$

Gravierender sind die Vorgänge in den Takten 5 und 6. Hier überrascht Purcell, indem er den Quartvorhalt $c^{1}$ nicht wie erwartet zu $h$, sondern stattdessen zu $b$ auflöst und gleichzeitig die Oberstimme von der Quinte des ersten Akkordes zur Sexte fortschreiten lässt, so dass ein Es-Dur-Sextakkord über dem gleichen Basston entsteht, was den Eindruck einer >doppelten Trugschlüssigkeitı erzeugt. Vergleichbare Wendungen sind zwar in der Vokalpolyphonie des späten 16. Jahrhunderts durchaus zu finden, im Rahmen eines homophonen, von Generalbassharmonik geprägten Satzes des ausgehenden 17. Jahrhunderts jedoch erscheint diese Akkordprogression eher befremdlich. Der erreichte Sextakkord sinkt in paralleler Chromatik zwei Stufen nach unten, gleitet quasi haltlos in die Tiefe, bis er vom Sekundakkord in Takt 7 wieder aufgefangen wird. Hieran schließt sich zum Abschluss des Vorspiels eine konventionelle Abkadenzierung nach c-Moll an,

3 Eine sogenannte `Heterolepsis`, die in dieser Zeit im freien Satz häufiger anzutreffen ist, hier aber nicht unerwähnt bleiben soll. 
die nach den Besonderheiten der vorangegangenen Takte die tonartliche Orientierung wiederherstellt.

Soweit die reine Beschreibung der Akkordfolgen; schwieriger ist allerdings eine plausible Erklärung und Einordnung dieser Befunde. Es macht durchaus nicht den Eindruck, als habe Purcell an irgendeiner Stelle beliebige Klänge aneinandergefügt; vielmehr wohnt dieser Harmonik eine Logik inne, die aufzudecken sich die folgende Analyse bemüht.

Versuchen wir zunächst eine traditionelle funktionstheoretische Analyse (Beispiel 1). Jedem Akkord ist eine Funktionsbezeichnung zugeordnet worden. Die Frage ist allerdings, ob sich die Abfolge der Funktionen auf die Kadenz als Grundmodell beziehen lässt und die Besonderheiten der Harmonik gefasst werden.
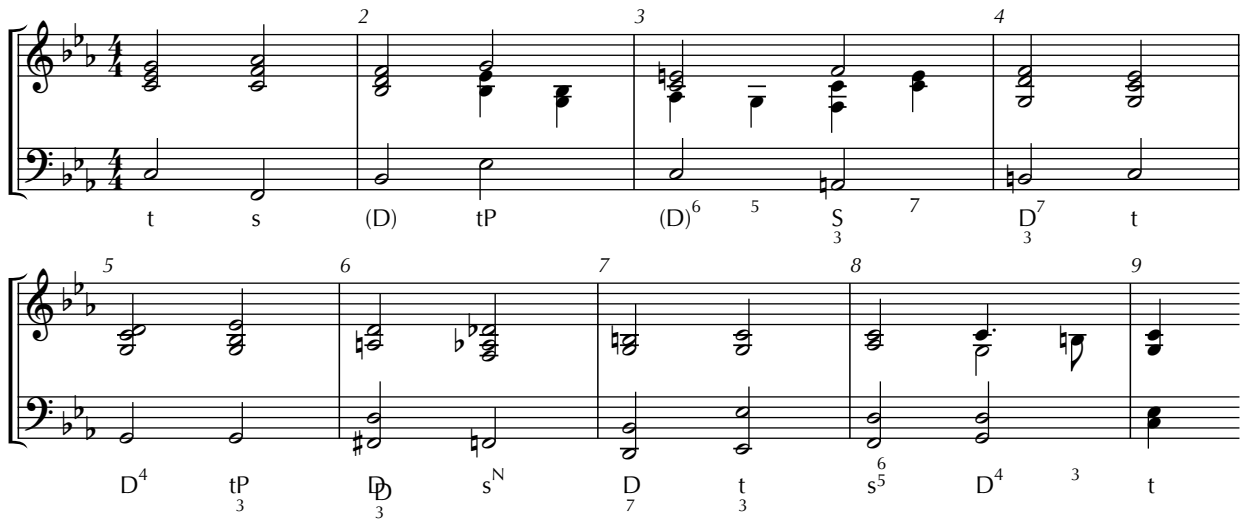

Beispiel 1

Tatsächlich liefert der gewählte Ansatz für die ersten vier Takten der Komposition relativ gute Ergebnisse. Die zweimal in Anschlag gebrachte Erklärung leittöniger Akkorde als Zwischendominanten ermöglicht es, den harmonischen Verlauf als eine einzige stark erweiterte Kadenz zu interpretieren. Als kleiner Schönheitsfehler bleibt, dass der Sextvorhalt und der Quintsextakkord in Takt 3 zwar bezeichnet, aber nicht eigentlich erklärt werden, zumal bei letzterem ein Widerspruch zwischen subdominantischer Funktion und Akkordform entsteht: Als Umkehrung eines kleinen Durseptakkords enthält sie strenggenommen die charakteristische Dissonanz der Dominante. ${ }^{4}$

Bezüglich der Takte 5 und 6 dagegen versagt die Funktionstheorie völlig. Schon die Abfolge Dominante - Tonikaparallele ist in Moll so nicht vorgesehen ${ }^{5}$, und die ungewöhnliche Auflösung des Quartvorhalts erfährt durch die Funktionsbezeichnungen ebenfalls keine befriedigende Erklärung, die über eine bloße Chiffrierung einzelner Klänge hinausgehen würde. Die Bezeichnung der nachfolgenden Sextakkorde als Tonika-

4 Den Quintsextakkord als Dominante einer elliptischen Kadenzierung nach B-Dur anzusehen, erscheint als unzulänglicher Notbehelf.

5 Die Bezeichnung `Tonikaparallele`suggeriert hier eine Stellvertreterfunktion der Tonika, die sich mit dem Hörerlebnis nicht deckt. 
parallele, Doppeldominante und Neapolitaner stellt sogar einen expliziten Widerspruch zum Kadenzdenken der Funktionstheorie dar, da die Vertreter von Subdominante und Dominante vertauscht erscheinen. Lediglich der Anschluss zum dominantischen Sekundakkord in Takt 7 wird verständlich, und dass die abschließende Abkadenzierung sich funktionstheoretisch einwandfrei erklären lässt, versteht sich von selbst.

Insgesamt zeigt sich also, dass uns die Funktionstheorie genau an jenen Stellen, um deren Erklärung wir eigentlich bemüht sind, im Stich lässt: Sie liefert zwar Benennungen der Akkorde, vermag sie aber nicht befriedigend zu erklären.

\section{III}

In einem zweiten Schritt soll nunmehr unter Bezug auf die Gesetze des Generalbasses, wie sie sich bereits kurz nach Purcells Tod, beispielsweise in der sogenannten >Oktavregel ${ }^{6}$ und den bei Corelli zu findenden Sequenzmodellen formuliert finden, ein historischer Ansatz an das Stück herangetragen werden.
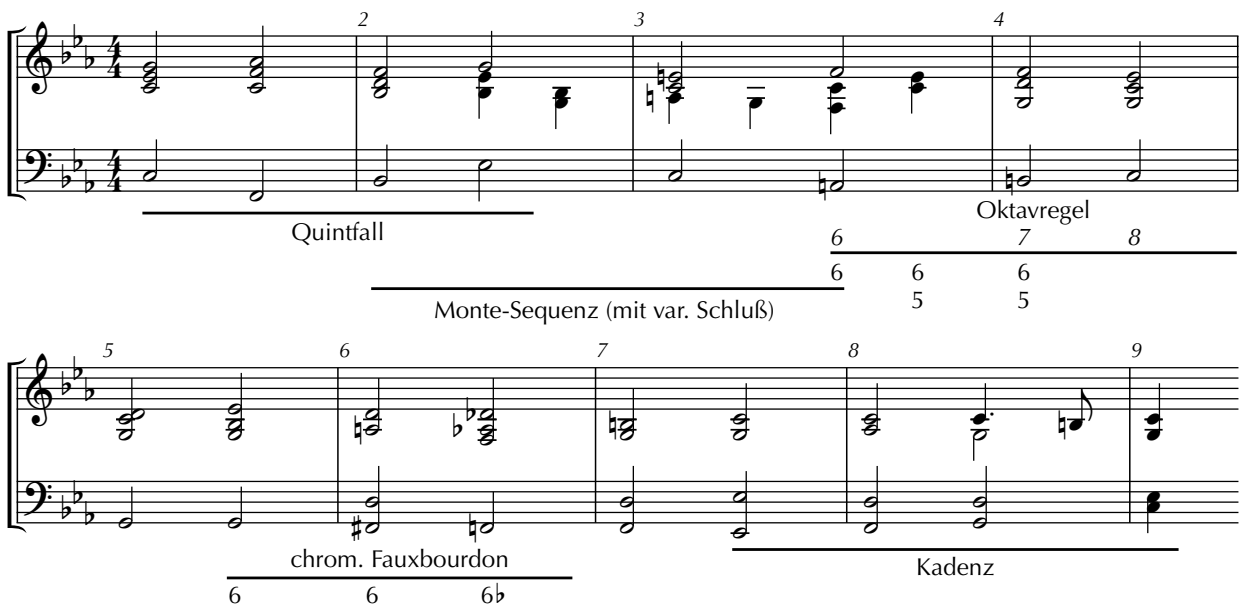

Beispiel 2

In der Tat wird man bei der Suche nach solchen Modellen durchaus fündig (Beispiel 2). Die ersten beiden Takte bilden eine typische Quintfallsequenz. Doch schon der nächste Takt stellt eine kleine Besonderheit dar: Die Takte 2 und 3 können als steigende `Monte`Sequenz ${ }^{7}$ betrachtet werden, die mit dem vorangegangenen Quintfall überlappt - im Ergebnis wird damit die Bassfolge des ersten Taktes wiederholt, die Verschiebung der ein-

6 So 1708 bei Francesco Gasparini, 1711 bei Antonio Bruschi.

7 Dieser Begriff wurde zwar erst gut fünfzig Jahre später von Joseph Riepel geprägt (1757), bezeichnet jedoch einen Sachverhalt, der aus Riepels Sicht bereits seit längerem zur Konvention gehörte. Die Anwendung von Riepels Begriff auf die Musik Purcells erscheint dadurch hinreichend gerechtfertigt. 
fachen Quintfälle wechselt lediglich die Richtung. Die Besonderheit hier ist nun, dass der letzte Akkord, der laut Montesequenz auf $f$ stehen müsste, durch einen Sextakkord auf a ersetzt wird. Dies wiederum dient der Überlappung mit einer weiteren modellhaften Wendung, bei der es sich um die Stufen 6 bis 8 einer Oktavregel handelt. In deren Zusammenhang ist auch der frei eintretende Quintsextakkord erklärlich, da er lediglich als klangliche Anreicherung des auf der 6. Stufe gebräuchlichen Sextakkordes erscheint, wie es die Oktavregel grundsätzlich zulässt. Die ersten vier Takte lassen sich somit fast lückenlos auf zeitgenössische Modelle zurückführen; lediglich der Sextvorhalt in Takt drei erscheint als klanglicher Zusatz, der sich nicht aus den genannten Modellen erklärt. ${ }^{8}$

Problematischer verhält es sich allerdings in den folgenden beiden Takten. So ist das Ersetzen der Quinte eines Grundakkords durch die Sexte zwar ein durchaus üblicher Vorgang (man denke an die 5-6-Konsekutive), und die Auflösung eines kadenzierenden Quartvorhalts zur kleinen statt großen Terz wird etwa von Angelo Berardi als eine Möglichkeit der Kadenzvermeidung beschrieben. ${ }^{9}$ Die Kombination beider Vorgänge in einer einzigen Akkordprogression allerdings erscheint doch eher ungewöhnlich. Die nachfolgende Kette von Sextakkorden bedeutet eine Modifikation der Oktavregel, die auf der vierten Bassstufe den Sekundakkord vorsieht, der hier erst im nächsten Takt erscheint. Der chromatische Bassgang mag Assoziationen an den Lamentobass in Verbindung mit dem Passus duriusculus aufkommen lassen, der allerdings normalerweise das obere Tetrachord ausfüllt und nicht, wie hier, das untere. Man könnte die Stelle als schromatischen Fauxbourdon` bezeichnen; angesichts dessen, dass der Fauxbourdon eigentlich eine diatonische Figur ist, würde dadurch ebenfalls eine Abweichung vom üblichen Modell kenntlich gemacht. Erst die letzten Takte gehorchen umstandslos barocken Kadenzierungsregeln.

Die Bezugnahme auf Modelle des Generalbasses bringt also durchaus etwas mehr Klarheit in die harmonische Progression. Es bleiben jedoch, wie gezeigt, immer noch gewisse Aspekte, vor allem bezüglich der Stimmführung und der Chromatik, die nicht befriedigend erklärt werden. Will man sich aber nicht damit zufrieden geben, diese Erscheinungen einfach als individuelle Varianten des blichen zu bezeichnen, sondern auch hier der inneren Logik und Motivation der Harmonik auf die Spur kommen, so wird es nötig, nach anderen Erklärungsmöglichkeiten zu forschen.

8 Das as der Viola ließe sich auch dadurch erklären, dass man nicht den Basston $c$, sondern das as als Grundton eines As-Dur-Dreiklangs mit Terz im Bass und hochalterierter Quinte (e statt es) ansieht, womit das bisherige Quintfallmodell in modifizierter Form weitergeführt würde. Das würde bedeuten, das nachfolgende $g$ der Viola als Durchgang aufzufassen. Dieser Interpretation steht aber entgegen, dass damit der konsonante C-Dur-Akkord, nicht der dissonante übermäßige Dreiklang zum strukturell höherwertigen Klang erklärt würde.

9 Im 3. Band seines Traktats Documenti armonici (Bologna 1687) zeigt Angelo Berardi unter dem Titel >Motivo di cadenza einige Beispiele einer solchen Auflösung des kadenzierenden Quartvorhalts zur kleinen Terz, die jeweils eine Modulation einleitet. Dabei bleibt allerdings, anders als im vorliegenden Stück von Purcell, die Quinte jeweils liegen oder springt zur Oktave, niemals jedoch zur Sexte. 
Zur Erhellung der Vorgänge soll deswegen in einem dritten Schritt die melodische Analyse auf dem Hintergrund spezifischer Skalen bzw. Skalenausschnitte hinzugenommen werden. Betrachten wir zu diesem Zweck zunächst den ersten Abschnitt nach Einsatz der Singstimme, also Takt 9 bis 16. Der homophone Akkordsatz bleibt erhalten, in ihn ist nun jedoch die Singstimme eingelassen, die in strenger Linearität den Tonraum von $c$ bis $c^{1}$ durchschreitet, wobei sie die Skala von c-Moll an mehreren Stellen um chromatische Zwischentöne erweitert. Dabei ergibt sich eine symmetrische Struktur: Der Oktavdurchgang besteht aus zwei gleich strukturierten Quartgängen mit einem chromatischen Zwischenschritt zwischen dem dritten und vierten Ton; dazu kommt das fis als beide Quartgänge chromatisch miteinander verbindender Ton, der gleichzeitig die Mitte der Oktave markiert. Diese Mischung von Diatonik und Chromatik macht den wesentlichen Charakter und Reiz der Melodiestimme aus.

Es handelt sich dabei eindeutig um eine Mittelstimme, die bisweilen, aber längst nicht immer, im instrumentalen Akkordsatz verdoppelt wird; dennoch ist diese Stimme, nicht etwa, wie man erwarten könnte, die Bass- oder Oberstimme, hauptsächlich Träger und Motivator der harmonischen Progression. Anders ließe sich etwa die Wendung nach gMoll in Takt 14 kaum verstehen. Dass der Akkordsatz und die Basslinie ansonsten durchaus konventionell angelegt sind, steht hierzu nicht im Widerspruch. Im übrigen legt die Strenge der Linearität der Gesangsstimme, welche die der Bassstimme noch übersteigt, es nahe, hierin in Art eines Cantus firmus die primäre Struktur für den Satz zu sehen.

Im weiteren Verlauf der Arie behält Purcell diese grundlegende Konzeption bei, unterwirft sie jedoch im Sinne der Gesamtgestaltung einem in sich geschlossenen Spannungsbogen. So entspricht der anfänglichen Aufwärtsbewegung ein Abstieg in den Takten 18-23, welche den zweiten Abschnitt des Gesangsteils bilden. Hier jedoch wird (noch) nicht die Oktave erneut durchschritten, statt dessen wendet sich die Harmonik nach f-Moll (was bereits durch den Variantwechsel nach C-Dur am Ende des Orchesterzwischenspiels in Takt 18 angekündigt wird), wodurch die Melodie etwa in der Mitte des zuvor aufgespannten Tonraums verharrt. So verbleibt genügend Restspannung, um den weiteren Verlauf der melodischen Entwicklung zu motivieren. Überraschend ist allerdings, dass Purcell dazu als Zwischenstufe die IV. statt, wie sonst üblich, die V. Stufe wählt, was im Widerspruch zu den Gepflogenheiten der Kadenzdisposition sowohl in älterer modaler als auch in generalbassbestimmter Musik steht.

Die genaue Gestaltung des Abstiegs knüpft durchaus an den vorigen Abschnitt an, indem die Stimme zunächst wieder den bekannten halbchromatischen Quartgang durchschreitet, also von $c^{1}$ über $b$, a und as bis $g$, wobei Purcell das Tempo der Tonfortschreitung in Takt 20 verändert, um den chromatischen Zwischenschritt unterzubringen. Vom Endpunkt des Quartgangs wäre es eigentlich nur ein simpler Sekundschritt zum Zielton $f$, jedoch zögert Purcell diesen hinaus, springt erst noch in einer expressiven Geste zum Ausgangspunkt $c^{1}$ zurück, von wo aus der neue Grundton in einem geradezu exaltierten Quintfall erreicht wird. Doch genügt diese Geste noch nicht, um den neuen Grundton zu etablieren - was durch die Harmonik, ein trugschlüssiges Des-Dur, absichtsvoll vereitelt wird. Dazu bedarf es erst noch eines eingeschobenen Leittons, der die Abwärtslinie 
weiter fortsetzt, und einer kompletten Kadenzformel (mit leittönig erhöhter IV. Stufe). Eine weitere Steigerung der Expressivität erreicht Purcell, indem er die meisten der abwärts gerichteten Fortschreitungen durch Vorhaltsbildungen hinauszögert.

Vom Endpunkt $f$ aus wendet sich die melodische Entwicklung ab Takt 25 wieder nach oben, um in Takt 29 mit es ${ }^{1}$ den Hochpunkt des Stücks zu erreichen - da dies harmonisch mit dem Wiedererreichen der Grundtonart c-Moll fast zusammenfällt (genauer mit ihrer Bestätigung durch eine einfache Kadenz ${ }^{10}$ ), kann hier vom Höhepunkt der Gesamtentwicklung gesprochen werden. Interessant ist wiederum eine zunächst rein melodische Betrachtung: Der Aufgang überschreitet zwar den vorherigen Hochpunkt um eine kleine Terz, fällt allerdings mit dem Umfang einer Septime etwas kleiner aus. Der halbchromatische Quartgang ist zwar nicht besonders exponiert, stellt aber in originaler Tonhöhe (von $g$ bis $c^{1}$, vgl. Takt 13-16, außerdem Umkehrung von Takt 18-21.2) sozusagen das Herzstück der Linie dar, das vom einleitenden $f$ vorbereitet und den beiden letzten Tönen überhöht wird. Ohne ein weiteres Zwischenspiel, das an diesem spannungsgeladenen Punkt nur stören würde, schließt sich ein letztes und endgültiges Zurückfallen der Melodie an, das vom Hochpunkt es ${ }^{1}$ aus fast den gesamten in der Arie verwendeten Tonraum noch einmal durchschreitet, um auf dem ursprünglichen Ausgangspunkt $c$ wieder zu enden. Die Verteilung der Chromatik entspricht hier weniger dem zuvor etablierten Muster als an den meisten anderen Stellen: Anstatt die chromatischen Zwischenschritte gleichmäßig auf den gesamten Abgang zu verteilen und so halbchromatische Quartgänge zu erzeugen, konzentriert Purcell sie auf die erste zweitaktige Phrase, die im Prinzip als chromatischer Leiterausschnitt von es ${ }^{1}$ bis $h$ anzusehen ist, bei dem jedoch das $c^{1}$ mit dem des ${ }^{1}$ vertauscht wurde - wohl um anschließend die hochexpressive verminderte Terz $d e s^{1}-h$ zu erzeugen, die schon im Orchestervorspiel in Takt 6/7 erscheint. Um die solcherart erzeugte Spannung etwas zu entschärfen, wird das $c^{1}$ wiederholt und zum Ausgangspunkt einer Weiterführung der Abwärtsbewegung durch die ganze Oktave gemacht. Diese verläuft nun jedoch streng diatonisch, was den resignativen Charakter dieser Endphrase unterstreicht - als lohne sich nach all der chromatischen Erregung zuvor keine weitere Chromatik mehr. Jedoch verzichtet Purcell auch in dieser Phrase nicht ganz auf expressive Mittel, da er wieder - wie bei der vorigen abwärts gerichteten Bewegung - die Möglichkeit zu Vorhaltsbildungen und entsprechenden Seufzerfiguren, gegen Ende auch Antizipationen nutzt. Die im Zusammenspiel mit der Bassstimme entstehende 7-6Konsekutive ruft trotz der Diatonik der Singstimme einige schmerzlich dissonante Klangeffekte hervor.

10 In der vorliegenden Ausgabe (hg. von Dennis Arundell, New York: Broude Brothers o.J.) steht in der 1. Violine und entsprechend im Continuopart ein $b$, was einen g-moll-Akkord ergäbe. Da diese Mollvariante der zu erwartenden Dominante G-Dur jedoch durch nichts motiviert ist, halte ich eine Verbesserung zu $h$ für zutreffend, was auch den mir zugänglichen Interpretationen entspricht. Wo genau dieser mutmaßliche Vorzeichenfehler verursacht wurde, ob bereits in Purcells Manuskript oder beim Verlag, war nicht zu ermitteln. 


\section{V}

Die stringente Satzstruktur in den Gesangsteilen lässt eine ähnliche den harmonischen Verlauf bestimmende Strukturstimme auch für das Orchestervorspiel vermuten. Da sie offensichtlich nicht als reale Stimme komponiert ist, müsste sie als eine Art Querschnitt aus dem Satz erschlossen werden. Der Akkordsatz erschiene dann nicht länger so schlicht, wie es zunächst den Anschein hat, und würde Momente innerer Bewegung aufweisen. In der Tat wird man bei dieser Betrachtungsweise des Vorspiels fündig, es lassen sich nämlich gleich zwei lineare Strukturstimmen isolieren (Beispiel 3).
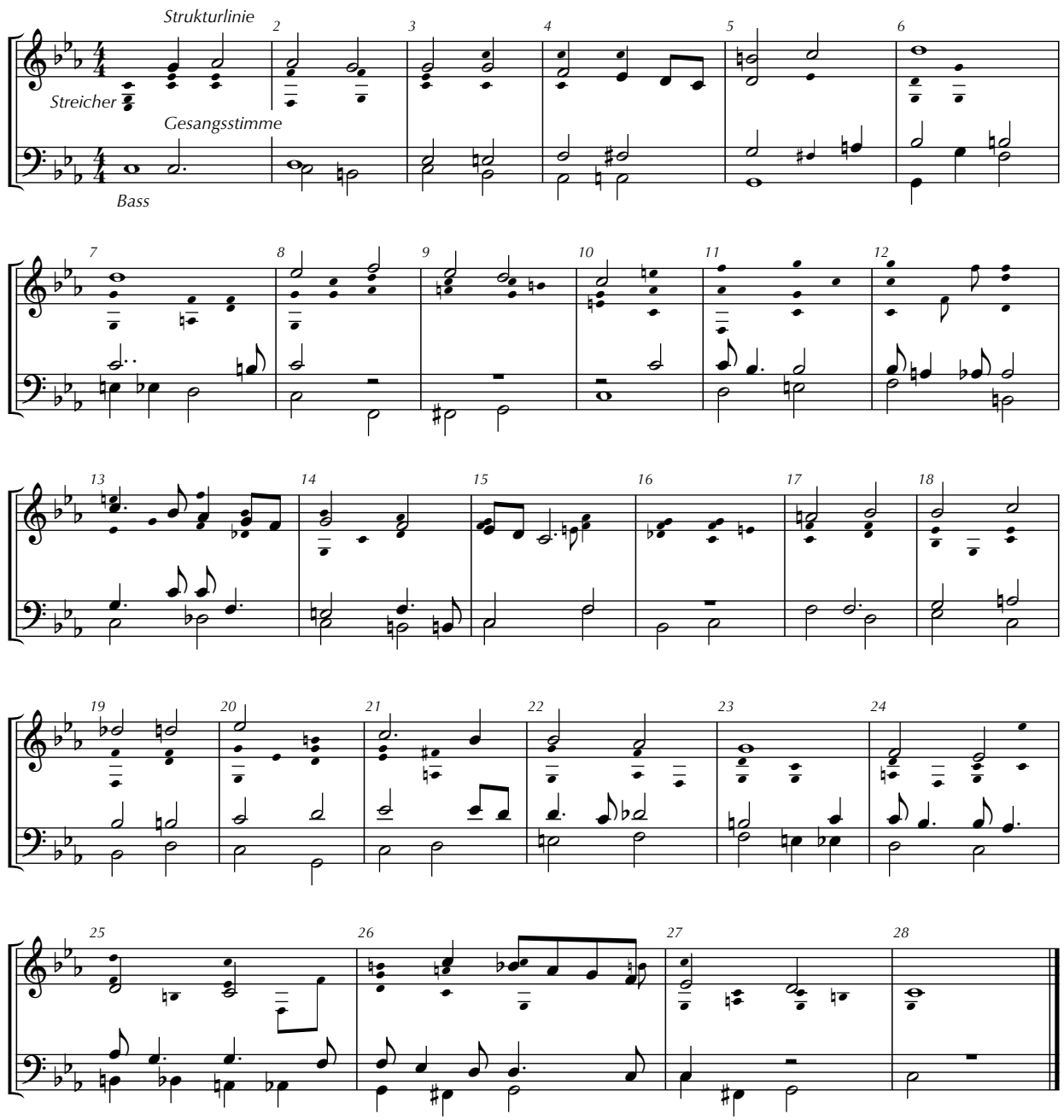

Beispiel 3 
Die erste Stimme beginnt auf $c^{1}$ (Bratschen) und schreitet dann durch 2. und 1. Violinen aufwärts bis $f^{1}$ in Takt 3, wobei sie, so wie später die Gesangsstimme, diesen Quartgang an einer Stelle chromatisch anreichert, nämlich durch das $e^{1}$. Diese melodische Progression erweist sich auch weiterhin als wesentlicher Grundbestandteil der melodischen Disposition beider Strukturstimmen. Nachdem nämlich die erste Stimme vier Schläge auf ihrem Spitzenton $f^{1}$ verweilt hat, sinkt sie ab Takt 4 entlang der gleichen, jetzt jedoch umgekehrten Struktur zurück zu $c^{1}$; hierbei wird sie lediglich durch eine nachfassende Wiederholung der Töne $e s^{1}$ und $d^{1}$ sowie ein aus kadentiellen Gründen eingefügtes $h$ erweitert, was an der Grundstruktur jedoch nichts ändert.

Die zweite Stimme beginnt ebenfalls auf $c^{1}$, sinkt nun aber in Gegenbewegung zur ersten abwärts, und zwar zunächst rein diatonisch entlang einer vollständigen natürlichen c-Moll-Skala. Lediglich aus instrumentatorischen Gründen wird diese Linie ab dem es von den 2. Violinen übernommen und dabei nach oben oktaviert. Interessant ist dabei auch die rhythmische Gestaltung: Nach einem jeweils relativ langen Verweilen auf den ersten beiden Tönen $c^{1}$ und $b$ wird sie im dritten Takt auf Viertel beschleunigt, um dann kurz vor Erreichen des Endtons wieder abgebremst zu werden. Dabei ist der Abstieg so angelegt, dass der Tiefpunkt $f$, nach dem die Stimme notabene nach oben springt, genau mit dem Hochpunkt $f^{1}$ der ersten Stimme zusammenfällt. Ähnlich einer Imitation der ersten Stimme verweilt die zweite ebenfalls vier volle Schläge auf dem erreichten $c^{1}$, um daran einen weiteren Abstieg anzuschließen, der nun den halbchromatischen Quartgang der Oberstimme übernimmt. Gleichzeitig imitiert die Stimme damit ihren eigenen Beginn, die so gewichtigen Töne $c^{1}$ und $b$. Der letzte Ton $g$ gerät dann zum finalen Halteton, lediglich durch eine Pendelbewegung zum as unterbrochen, die zwar durch die Kadenzbildung bedingt ist, jedoch gleichzeitig wie ein Echo der entsprechenden Bewegung der Oberstimme in Takt 5/6 wirkt.

Aus diesen beiden Stimmen lässt sich im Prinzip die gesamte harmonische Progression der Stelle lückenlos erklären. Vor allem werden vor diesem Hintergrund die bisher als eigenartig und schwer erklärbar empfundenen Stellen logisch herleitbar: Sowohl der freie Sextvorhalt als auch die dissonierende Quinte in Takt 3 sind schlichtweg Teil der unteren Strukturstimme und damit nicht nur klangliche Zutat, sondern essentieller Bestandteil der musikalischen Struktur. Die irreguläre Auflösung des Quartvorhalts in Takt 5 erscheint durch den imitatorischen Bezug zum Anfang geradezu zwingend; die Progression zum Sextakkord ist direkte Folge der Gegenbewegung der beiden Stimmen, ebenso wie die anschließende Sextakkordkette aus den chromatischen Quartparallelen der beiden Stimmen hervorgeht. Was nicht in den Strukturstimmen ausgedrückt ist oder zwingend aus ihnen hervorgeht, entspricht harmonischer Konvention, so etwa der anfängliche Quintfall oder die Schlusskadenz.

Da das Vorspiel wie gesehen von der Interaktion zweier Strukturstimmen bestimmt wird, liegt es nahe, auch im Hauptteil der Arie das Vorhandensein einer zweiten Strukturstimme neben der Gesangsstimme anzunehmen. Erst durch den Verlauf beider Linien ent- 
steht das Stimmengerüst, das für die Harmonik ausschlaggebend ist (Beispiel 4). Diese zweite Stimme wechselt wie im Orchestervorspiel zwischen den ausnotierten Orchesterstimmen hin und her, was auch hier an ihrer strukturellen Relevanz hinsichtlich der Harmonik nichts ändert.
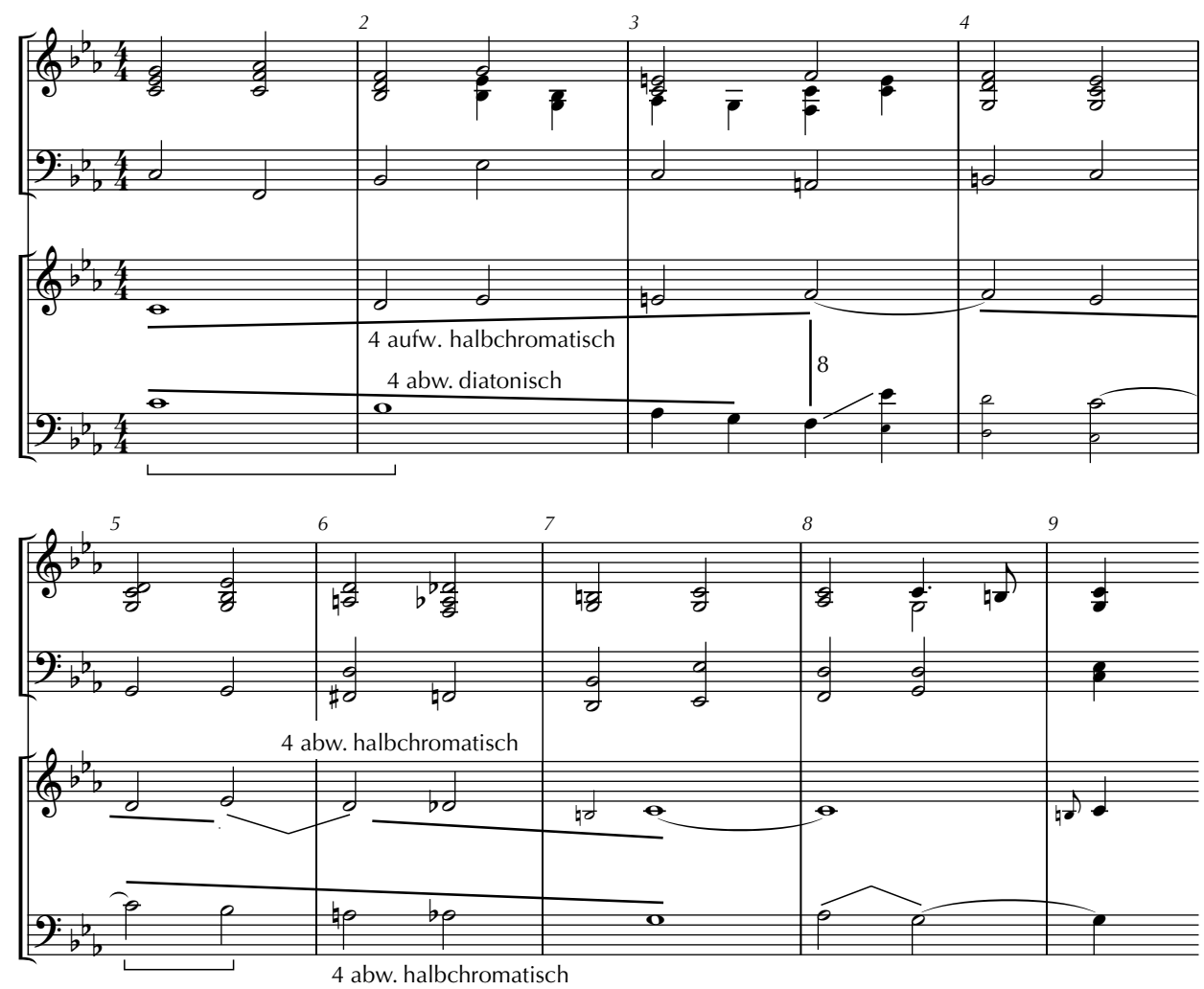

Beispiel 4

Relativ unspektakulär und rein diatonisch nimmt sich diese zweite Stimme im ersten Abschnitt des Gesangsteils Takt 9-16 aus. Sie ist zunächst Oberstimme in den 1. Violinen, wo sie den Schritt $g^{1}-a s^{1}$ vom Beginn des Vorspiels zitiert, im Gegensatz hierzu jedoch wieder schrittweise zurücksinkt. In Takt 11, wo die 1. Violinen zum $c^{2}$ springen, setzt sich dieser Abstieg in den 2. Violinen fort, und zwar im Prinzip halbtaktig bis zum Erreichen des $d^{1}$ in Takt 13 (von der vorausgreifenden Auszierung in Takt 12 abgesehen). In den folgenden Takten ist die Linie nicht ganz so offensichtlich, man kann jedoch durchaus die 1. Violinstimme als oktavierte Beantwortung des Abstiegs in Gegenbewegung ansehen - von $h^{1}$ über $c^{2}$ und ein sehr lang gehaltenes $d^{2}$ zunächst zu $e s^{2}$, im Rahmen der eingeschobenen Kadenz sogar bis $f^{2}$, wovon die Stimme linear bis $c^{2}$ zurücksinkt. 
Zu Beginn des zweiten Gesangsteils ist erstmals die Bassstimme als wesentlicher linearer Widerpart der Gesangslinie zu sehen; ihr Gang von c zu $f$ setzt eindeutig den Wechsel zu f-Moll in Gang. Danach verliert sich jedoch die Linearität dieser Stimme, die stattdessen hauptsächlich um $c$ kreist. Zu beobachten ist dagegen ab Takt 21 ein diatonischer Abstieg in den 2. Violinen, der in Takt 23 von den Bratschen fortgesetzt wird. Interessanterweise ergibt sich dabei wie im Vorspiel eine rein diatonische c-MollTonleiter, obwohl doch der Abschnitt eigentlich in f-Moll steht. Dies erklärt gleichzeitig das merkwürdige es ${ }^{1}$ in den Bratschen zu Beginn von Takt 23, das sich so ungewöhnlich mit dem Quartvorhaltsklang reibt, den die übrigen Stimmen bilden. Dieser Ton erklärt sich nicht als eine betonte Wechselnote, wie die Stimmenaufteilung es zunächst vermuten lässt; vielmehr ist er Teil einer übergeordneten linearen Struktur mit formal und thematisch relevantem Bezug, wodurch der Klang eher verständlich wird. ${ }^{11}$

Ein ähnlicher Effekt ergibt sich kurz vor Schluss der Arie in Takt 34, wo eine entsprechend rätselhafte Wechselnote $b^{1}$ sich bei näherem Hinsehen ebenfalls als Teil der natürlichen c-Moll-Leiter ${ }^{12}$ erweist, die vom $c^{2}$ der 1 . Violinen ausgehend zunächst durch die 2. Violinen bis zum es $s^{1}$ läuft, um in Takt 35 wieder von den 1. Violinen übernommen und zu Ende geführt zu werden.

Weitere lineare Strukturen finden sich ab Takt 24: Das in den 1. Violinen liegende $g^{1}$ schreitet zum $a^{1}$ fort und leitet damit im Wesentlichen eine Überterzung der Singstimme ein (bis auf die rhythmische Verschiebung in Takt 25/26, die wesentlich die Tonartverschiebung nach B-Dur einleitet). Man könnte sogar das $f^{1}$ der 2 . Violinen aus Takt 23 noch hinzurechnen und in den folgenden Takten eine unvollständige F-Dur-Skala sehen, die oberhalb der Quinte chromatisch angereichert wird. Entscheidender ist allerdings, dass hier wiederum ein halbchromatischer Quartgang (zwischen $b^{1}$ und $e s^{2}$ ) entsteht, der dadurch noch betont wird, dass die Aufwärtsbewegung nach dem $e s^{2}$ abrupt abbricht - einen Takt, bevor die Singstimme ebenfalls es erreicht. Aus der Parallelführung der beiden halbchromatischen Quartgänge in 1. Violine und Singstimme ergibt sich auch die effektvolle Wendung nach b-Moll in Takt 27, die - das ist das eigentlich Überraschende - harmonisch folgenlos bleibt, da im Zuge der fortgesetzten Chromatik die Tonart nach c-Moll verschoben wird. Im übrigen stellen die letzten vier Töne des Quartgangs der Violinen, die Töne $c^{1}$ bis $e s^{2}$, einen direkten Bezug zum Vorspiel her, da sie den oktavversetzten Krebs der Takte 4.3 bis 7 (ohne das eingeschobene $h$ ) bilden. ${ }^{13}$

11 Die gleiche lineare Struktur erklärt im übrigen auch, warum in Takt 23 in den Bratschen zu Recht nicht des (wie es der gleichzeitig ablaufenden f-Moll-Kadenz entsprechen würde), sondern $d$ steht.

12 Der dem $b^{1}$ folgende Ton sollte folgerichtig $a s^{1}$ heißen, was ebenfalls der beobachteten Praxis entspricht.

13 Das hartnäckige Erscheinen des Tons des, der in verschiedenen harmonischen Zusammenhängen sowohl im Vorspiel als auch im Mittelteil der Arie auftaucht, fordert eine Erklärung: in Takt 6 als neapolitanische Sexte über der IV. Stufe, in Takt 21 als Basston eines Trugschlusses in f-Moll, dann im Rahmen der Kadenz auf $f$ in Takt 24 und schließlich als Mollterz des anvisierten b-Moll-Dreiklangs in Takt 27. Ein dermaßen gehäuftes Auftreten legt eine gezielte kompositorische Absicht nahe: Die kleine Sekunde über dem Grundton ist als Ausdrucksmittel des Leides und des Schmerzes zu Purcells Zeit verbreitet und deutet, im Textzusammenhang gesehen, auf die Schmerzhaftigkeit des Aufsteigens des Cold Genius hin. Unter diesem Gesichtspunkt kann man durchaus annehmen, 
Auf die Überterzung folgt im nächsten Abschnitt zunächst eine Unterterzung, allerdings oktavversetzt und ohne die Vorhalte und Vertauschungen, so dass real Sexten erklingen. Beim Wiederaufgreifen des $C^{1}$ durch die Singstimme bleibt die Gegenstimme allerdings liegen, so dass aus den Unterterzen bzw. Obersexten nun Unterquarten bzw. Oberquinten werden - nur durch ausgiebigen Gebrauch der Vorhalte vermeidet Purcell hier Quintparallelen. Ab Ende von Takt 32 wandert die lineare Stimme durch 2. Violinen und Bratschen. Somit ergibt sich wiederum eine vollständige c-Moll-Skala, so dass nun in diesem letzten Abschnitt gleich drei abwärts sinkende c-Moll-Leitern miteinander verschränkt werden. Dies zeigt einmal mehr die thematische Relevanz dieser Skala und weist deutlich darauf hin, dass sie auch bei ihrem vorherigen Erscheinen im Vorspiel und dem zweiten Gesangsabschnitt keinesfalls als zufällige Erscheinung, sondern als bewusst komponierte Struktur anzusehen ist.

Auch das Element der Chromatik ist nach Takt 30 nicht verschwunden, sondern findet sich an prominenter Stelle wieder: Zwischen Takt 31 und 33 sinkt die Bassstimme von $c$ aus chromatisch abwärts, füllt den an anderer Stelle etablierten Quartraum chromatisch aus und erweitert ihn sogar um Fis. Damit zitiert die Bassstimme einerseits eine für Purcell bereits konventionelle Figur, den Lamentobass, andererseits stellt sie eine Verbindung zum früher verwendeten halbchromatischen Quartgang her, der nun zur vollen Chromatik aufgefüllt wird.

\section{VII}

Im Lichte der vorangegangenen Analyse erscheint die Harmonik als Ergänzung einer zweistimmigen linearen Struktur. Lediglich eine der beiden Stimmen manifestiert sich in Gestalt der Singstimme, die andere Stimme bleibt in gleicher Weise implizit, wie dies im Vorspiel für beide Stimmen der Fall war. Auffällig ist hierbei, dass diese Strukturstimmen zwar sämtliche Ober- und Mittelstimmen, aber nur selten die Bassstimme berühren. Dies widerspricht der Auffassung, die harmonischen Progressionen des Generalbasszeitalters seien grundsätzlich vom Bass aus konzipiert. Zwar stellt der Bass auch hier das Fundament der Akkorde dar, und Purcell legt durchaus auch Wert auf eine nachvollziehbare und melodisch sinnvolle Bassstimme. Sie allein konstituiert aber noch nicht die ganze Harmonik, vielmehr wird diese erst aus dem Zusammenspiel von Strukturstimmen und Bassverlauf hergestellt.

Hierdurch ergibt sich eine merkwürdige Konkurrenzsituation zwischen reinem Stimmendenken, das sich aus der Tradition des Intervallsatzes speist, und einem Verständnis von Generalbass als Grundlage aller harmonischen Strukturen, wie es sich zu Purcells Zeit allmählich durchsetzte. Darin spiegelt sich Purcells historische Situation wider, die vom Übergang zwischen beiden Denkweisen bestimmt ist. Purcell scheint diesen Wandel nicht nur wahrgenommen, sondern kompositorisch regelrecht instrumentalisiert zu haben: Anstatt Stimmverläufe und Generalbass so miteinander zu harmonisieren, dass sie sich gegenseitig stützen und erklären, erzeugt er bewusst Gegensätze zwischen bei-

dass die ungewöhnliche Kadenzstufe $f$ von Purcell hauptsächlich gewählt wurde, um ein möglichst häufiges und unterschiedliches Erscheinen des Tones des zu ermöglichen. 
den Ebenen, indem er die Stimmverläufe so wählt, dass sich andere harmonische Fortschreitungen ergeben, als dies in einer reinen Generalbassharmonik der Fall wäre.

Fragt man nach der Motivation für diesen bewusst herbeigeführten Gegensatz, so wird es nötig, die inhaltliche Dimension dieser Opernarie zu berücksichtigen. Sie stellt ja die Antwort des Geistes auf den Ruf Cupidos dar, durch welchen der Geist gezwungen wird, sich zu erheben, obwohl er eigentlich sofort wieder in eisiger Erstarrung versinken möchte. Diese dramatische Situation stellt die melodische Linie der Singstimme bildlich dar, wobei sie dramaturgisch ausgesprochen geschickt disponiert ist: Einem initialen Aufstieg als erstem Erscheinen des Geistes, das durch die eingefügte Chromatik als besonders mühsam und widerstrebend gekennzeichnet wird, folgt ein expressiv aufgeladenes Absinken als Klage (»See'st thou not how stiff ... I am? «), dann ein erneutes, gesteigertes Aufsteigen zum Höhepunkt, von dem aus die Linie zunächst in erneuter expressiver Klage, dann zunehmend resignierend zurückfällt und am Ende, wo schon kurz vor dem Schlusston der Achtelpuls verlassen wird, regelrecht versinkt. Eindrücklicher ließe sich das "freeze to death«, um das der Geist hier bittet, musikalisch kaum umsetzen.

Bei der Gestaltung des diastematischen Verlaufs im Verhältnis zur geschilderten Szene belässt es Purcell nicht bei einer äußerlichen Ähnlichkeit, sondern nimmt bestimmte Zuordnungen vor: Dem mühsamen, erzwungenen Aufstieg entspricht der steigende halbchromatische Quartgang, das resignierte Zurücksinken am Ende erhält die Form der absteigenden c-Moll-Skala. Gleich zu Beginn des Vorspiels werden beide Hauptgestalten kontrapunktisch gegeneinander gesetzt; es liegt durchaus nahe, die aufsteigende Linie als Kontrasubjekt zur absteigenden Mollskala zu sehen. Als Mischform schließt sich in beiden Strukturstimmen der absteigende halbchromatische Quartgang an, der den Gegensatz zwischen beiden Momenten in sich vereint und damit die Klage des Geistes über seine unwillkommene Situation zum Ausdruck bringt.

Der die Arie durchziehende Gegensatz zwischen Erheben und Zurücksinken ist also von Anfang an präsent und zieht sich durch die ganze Arie. Erst in den letzten acht Takten löst sich dieser Gegensatz auf: Die aufsteigenden Linien verschwinden, die diatonische c-Moll-Skala überwiegt und tritt ungewöhnlich gehäuft auf, die Chromatik nimmt die reguläre Form des Lamentobasses an, den man im Vorspiel noch allenfalls assoziieren konnte. Entsprechend erscheint auch die Harmonik zu einer regelmäßigen 7-6-Konsekutive mit anschließender Kadenz geglättet. Der quälende Widerstreit entgegengesetzter Elemente endet: Der Geist sinkt in die eisige Kälte zurück, aus der Cupido ihn emporsteigen ließ.

\section{Literatur}

Adams, Martin (1995), Henry Purcell: The Origins and Development of His Musical Style, Cambridge: Cambridge University Press.

Berardi, Angelo (1687), Documenti armonici, Bologna. http://www.musica-antica.info/ berardi/documenti/documenti_home.php

Burden, Michael (1995), The Purcell Companion, London: Faber \& Faber. 
Keates, Jonathan (1995), Purcell. A Biography, London: Chatto \& Windus.

Lester, Joel (1992), Compositional Theory in the Eighteenth Century, Cambridge MA/London: Harvard University Press.

Price, Curtis A. (1984), Purcell and the London Stage, Cambridge: Cambridge University Press.

— (1995a), Purcell Studies, Cambridge: Cambridge University Press. (1995b), King Arthur: the Music, Beiheft zur CD Purcell: King Arthur, Les Arts Florissants, Paris: Erato.

Quervain, Fritz de (1935), Der Chorstil Henry Purcells. Studien zu seinen Anthems, Bern/Leipzig: Haupt.

Riepel, Joseph (1752-1768), Anfangsgründe zur musicalischen Setzkunst, Bd. 3: „Gründliche Erklärung der Tonordnung insbesondere«, Frankfurt/Leipzig. 